\title{
UMA ANÁLISE COMPARATIVA SOBRE A RENTABILIDADE DE INVESTIMENTOS EM BITCOINS
}

\section{A COMPARATIVE ANALYSIS ON THE PROFITABILITY OF INVESTMENTS IN BITCOINS}

\section{UN ANÁLISIS COMPARATIVO SOBRE LA RENTABILIDAD DE LAS INVERSIONES EN BITCOINS}

\section{Rubens Teixeira da Silva}

Mestre em Desenvolvimento Local pela UNISUAM/RJ. MBA em Gestão Financeira, Controladoria e Auditoria pela UCB/RJ. Graduação em Administração pela UNIGAMA/RJ e em Gestão em Controladoria e Recursos Humanos pela UNIFOA/RJ.

rubestrjrj@gmail.com

http://orcid.org/0000-0002-2998-6411

Maria Geralda de Miranda

Pós doutora em Políticas Públicas e Formação Humana pela Universidade do Estado do Rio de Janeiro (UERJ). Pesquisadora do Programa de Pós-graduação em Desenvolvimento Local do Centro Universitário Augusto Motta, UNISUAM, Rio de Janeiro, RJ.

mgeraldamiranda@gmail.com

https://orcid.org/0000-0002-2461-7414

Kátia Eliane Santos Avelar

Doutora em Ciências pela Universidade Federal do Rio de Janeiro, UFRJ. Docente e Pesquisadora do Programa de Pós-graduação em Desenvolvimento Local do Centro Universitário Augusto Motta, UNISUAM, Rio de Janeiro, RJ.

katia.avelar@gmail.com

https://orcid.org/0000-0002-7883-9442

\section{Editor Científico: José Edson Lara}

Organização Comitê Científico

Double Blind Review pelo SEER/OJS

Recebido em 09.11.2018

Aprovado em 08.10.2020 


\section{Resumo}

Objetivo do estudo: O objetivo deste estudo foi analisar a volatilidade e a rentabilidade do bitcoin, em comparação com três carteiras de ativos de ações que apresentaram excelente grau de avaliação ao longo do período analisado.

Metodologia/abordagem: $O$ estudo reuniu informações tratadas por meio do método quantitativo e de uma pesquisa de campo. Decidiu-se utilizar como técnica o modelo autoregressivo $\operatorname{AR}(p)$, buscando-se o retorno por meio da média incondicional $\operatorname{AR}(p)$ e da variância incondicional $\operatorname{ARCH}(p)$ ou $\operatorname{EGARCH}(p, q)$.

Originalidade/Relevância: No Brasil, existem diferentes tipos de investimentos. Para alguns investidores, as moedas eletrônicas se tornaram um tipo de investimento comparável ao ouro ou dólar. Este estudo pretendeu mostrar para os investidores como o bitcoin pode ser utilizado como uma alternativa para investimento.

Principais resultados: Desde o início do ano de 2017, verificou-se que houve um aumento do valor do bitcoin, alcançando o valor de USD13.411,00 em 31 de dezembro de 2017. A supervalorização repentina e o aumento no número de usuários despertaram os analistas, Estados e investidores a observar a questão da volatilidade dessa moeda. Foram analisados o período de 2015 a 2017 para o cálculo da volatilidade dos ativos e do bitcoin.

Contribuições teóricas/metodológicas: Os resultados obtidos revelaram o grau de conhecimento dos participantes em relação a aplicações financeiras, um possível perfil para investidores em bitcoin, a rentabilidade e a volatilidade dos ativos comparados, possibilitando a comparação do bitcoin com outros ativos de renda variável.

Palavras-chave: Bitcoin, volatilidade, rentabilidade, ativos de renda variável.

\section{Abstract}

Objective of the study: The objective of this study was to analyze the volatility and profitability of bitcoin, in comparison with three portfolios of stock assets that showed an excellent degree of valuation over the analyzed period.

Methodology / approach: The study gathered information treated using the quantitative method and a field survey. It was decided to use the AR (p) autoregressive model as a technique, looking for the return by means of the unconditional average AR (p) and the unconditional variance ARCH (p) or EGARCH (p, q).

Originality / Relevance: In Brazil, there are different types of investments. For some investors, electronic currencies have become a type of investment comparable to gold or the dollar. This study aimed to show investors how bitcoin can be used as an investment alternative.

Main results: Since the beginning of 2017, there has been an increase in the value of bitcoin, reaching the value of USD13,411.00 on December 31, 2017. The sudden overvaluation and the increase in the number of users have awakened analysts, states and investors to look at the currency's volatility issue. The period from 2015 to 2017 was analyzed to calculate the volatility of assets and bitcoin. 
Theoretical / methodological contributions: The results obtained revealed the degree of knowledge of the participants in relation to financial applications, a possible profile for bitcoin investors, the profitability and volatility of the compared assets, enabling the comparison of bitcoin with other variable income assets.

Keywords: Bitcoin, volatility, profitability, variable income assets.

\section{Resumen}

Objetivo del estudio: El objetivo de este estudio fue analizar la volatilidad y rentabilidad de bitcoin, en comparación con tres carteras de activos bursátiles que mostraron un excelente grado de valoración durante el período analizado.

Metodología / enfoque: El estudio recopiló información tratada mediante el método cuantitativo y una encuesta de campo. Se decidió utilizar como técnica el modelo autorregresivo AR (p), buscando el retorno mediante el AR promedio incondicional (p) y la varianza incondicional ARCH (p) o EGARCH (p, q).

Originalidad / Relevancia: En Brasil, existen diferentes tipos de inversiones. Para algunos inversores, las monedas electrónicas se han convertido en un tipo de inversión comparable al oro o al dólar. Este estudio tuvo como objetivo mostrar a los inversores cómo se puede utilizar bitcoin como alternativa de inversión.

Principales resultados: Desde principios de 2017, se ha producido un incremento en el valor de bitcoin, alcanzando el valor de USD13.411,00 al 31 de diciembre de 2017. Ha despertado la sobrevaloración repentina y el aumento en el número de usuarios. analistas, estados e inversores para analizar el problema de la volatilidad de la moneda. Se analizó el período de 2015 a 2017 para calcular la volatilidad de los activos y bitcoin.

Aportes teóricos / metodológicos: Los resultados obtenidos revelaron el grado de conocimiento de los participantes en relación a las aplicaciones financieras, un posible perfil de inversionistas de bitcoins, la rentabilidad y volatilidad de los activos comparados, permitiendo la comparación de bitcoins con otros activos de renta variable.

Palabras clave: Bitcoin, volatilidad, rentabilidad, activos de renta variable.

\section{INTRODUÇÃO}

A busca por retornos numerosos motiva os investidores a empregar valores relevantes em algum tipo de aplicação financeira, outros preferem investir em ativos que minimizem o risco. A experiência em se investir, em alguns casos não leva em consideração a segurança, mas sim os valores do resgate. A experiência do passado revela que cenários futuros podem por natureza sofrer modificações. A inflação pode baixar e o nível da atividade econômica 
subir. Contudo, a instabilidade do ambiente econômico em que estão inseridos os projetos e investimentos pode ser constante.

No Brasil, existem diferentes tipos de investimentos, dentre eles o mercado imobiliário, a poupança, CDB (Certificado de depósito bancário), debêntures, obras de arte e antiguidades, ouro, poupança, previdência privada, títulos públicos, ações, dentre outros. Para muitos, as moedas eletrônicas se tornaram um tipo de investimento, tendo repercuções como ouro ou dólar. Embora as técnicas sejam basicamente parecidas para um investimento onde se necessita avaliar a rentabilidade, a estabilidade, o cenário, os riscos, dentre outras medidas; apesar das incertezas sobre a moeda eletrônica, verifica-se que o bitcoin vem crescendo em número cada vez maior de usuários. Entretanto, o investimento mais procurado por muitos brasileiros são os títulos do governo, devido ao fato de ser seguro. Com o aparecimento da crise em 2008, alguns investimentos tiveram perda de consumidores e verificou-se um aumento significativo nas transações de bitcoin. Diante desta realidade surge a seguinte pergunta: de fato os investidores buscam segurança ou lucro?

O objetivo principal desta pesquisa foi analisar a volatilidade para um investimento com bitcoin em comparação com outros ativos de renda variável de 3 diferentes carteiras de ações. Para isso, o estudo utilizou uma pesquisa de campo, com visita a uma mineradora de bitcoin e participação em palestras sobre a moeda, decidiu-se abordar o método quantitativo para comparar o retorno e a volatilidade do bitcoin com uma carteira diversificada de ativos que apresentaram excelente avaliação para o período. Verificou-se que todos os tipos de investimentos estão sujeitos a riscos, podendo este advir de fatores políticos, econômicos ou naturais. Decidiu-se considerar a capacidade de investidores estimarem as consequiências da inflação sobre um projeto de aplicação financeira e seus efeitos em longo prazo.

\section{REFERENCIAL TEÓRICO}

\subsection{Estudos recentes sobre bitcoin relacionados à área financeira}

Correia, Alencar e Amilton (2017) verificaram que a variação dos retornos do Bitcoin se relaciona apenas com o fator de risco investimento (CMA), o que indica que empresas com elevados níveis de investimento tendem a impulsionar os retornos do Bitcoin. Conforme descrito por Mesquita (2017) em uma análise sobre uma perspectiva pós Keynesiana,

verificou-se que a moeda pode ter um alto prêmio de liquidez relativa, mas seu 
comportamento como ativo, com o qual se pode especular, a torna vulnerável a ciclos. Uma das características da moeda é que sua liquidez é de natureza neutra em relação ao ciclo econômico.

Segundo Mesquita (2017), analisando a moeda no setor financeiro, verificou-se o potencial de seu impacto no setor, com novos modelos concorrentes, possibilitando a intermediação de taxas do sistema financeiro tradicional que poderão ficar mais baixas. Luis (2016) fez uma análise por meio do modelo de Markowitz (1952), citando o bitcoin como um tipo de ativo, verificando que a população brasileira opta em investir suas aplicações na poupança, e observando que esta não é a melhor alternativa de investimento. Constatou que é possível formular carteiras de acordo com o perfil dos investidores, com o interesse de maximizar os rendimentos de cada indivíduo.

Segundo Barreiros (2016) em um estudo sobre análise de comercio internacional, verificou que a tecnologia por detrás da bitcoin poderá mitigar os efeitos de eventuais crises financeiras, na medida em que a blockchain permitir aos reguladores visualizarem automaticamente uma ampla gama de eventos financeiros, incluindo detalhes comerciais, métricas de risco de contraparte e exposição a entidades de referência de forma transparente e automática para além de servir de "moeda de refúgio" ter a sua convertibilidade aceita.

\subsection{Investimentos e bitcoin}

Com a retomada do crescimento econômico em 2017, iniciou-se, novamente a procura por investimentos. De acordo com o Tesouro Nacional (2017), 23,2\% das vendas no tesouro no mês de março corresponderam a títulos com vencimentos acima de 10 anos. As vendas de títulos com prazo entre 5 e 10 anos representaram $15 \%$ e as com prazo entre 1 e 5 anos, 61,8\% do total.

Os investimentos em renda fixa como a poupança e o tesouro direto, permitem maior segurança quando se refere a um período longo, sendo estes os principais no interesse dos brasileiros. Segundo o Banco Central do Brasil (2016), 63\% da população optam por aplicar dinheiro na caderneta de poupança. De acordo com Rossetti (2007), a alternativa do investidor ao escolher a composição de sua carteira de investimento depende do retorno esperado e de seu risco.

Alguns tradicionalistas se renderão aos títulos habituais, se porventura surgir uma nova forma de investimento, poderão ficar hesitados neste novo projeto. Markowitz (1952) 
afirma que, para determinado investimento, deve existir uma carteira que maximize o retorno esperado e minimize a variância, sendo essa carteira indicada ao potencial investidor.

Segundo Bourdeaux; Ness (2007), o risco de uma carteira com diversos ativos é menor que o de um único ativo que ofereça o mesmo retorno da carteira. Este pensamento demonstra a forma de muitos investidores visualizarem no mercado de ações a possibilidade de investimentos com diversas carteiras que minimizem perdas e riscos, não os deixando livres das constantes mudanças que o mercado de ações pode sofrer, deixando-os, contudo, com diversas possibilidades que poderão minimizar os riscos.

Nota-se, contudo, a preocupação com o perfil do investidor para cada tipo de carteira de investimentos, até mesmo para o bitcoin. Alguns irão preferir a segurança, outros uma melhor rentabilidade. Por outro lado, o mercado de moedas eletrônicas já demonstra grande número de moedas, sendo o total de 1339, conforme o site Cryptocurrency Market Capitalizations (2017). Não obstante a isto, a principal bolsa de valores da Austrália anunciou que se tornará o primeiro mercado de ações a utilizar a tecnologia existente no bitcoin, substituindo o atual sistema de compensação pela tecnologia blockchain (Américo, 2017). Ademais, outros mercados financeiros como a bolsa de Chicago nos EUA anunciaram que começarão a operar transações com bitcoin ainda em dezembro de 2017 (Oliveira, 2017).

\section{METODOLOGIA}

Para a realização deste estudo, foram utilizados dois tipos de pesquisa, uma pesquisa de campo e a quantitativa. Na primeira, houve a participação em palestras em uma empresa de mineração de bitcoin, localizada na cidade do Rio de Janeiro. Foram entrevistadas 40 pessoas participantes de investimentos com bitcoins, dentre elas indivíduos do sexo masculino e feminino, entre a idade de 18 a 50 anos. Houve ainda a participação de 40 pessoas da população, pertencentes às classes $\mathrm{A}, \mathrm{B}$ e $\mathrm{C}$, indivíduos do sexo masculino e feminino, entre a idade de 18 a 50 anos, todos com nível superior e com o perfil para investimentos, sendo estes indivíduos residentes nos bairros de Bonsucesso, Centro e Flamengo, na cidade do Rio de Janeiro.

Para este tipo de pesquisa foram desenvolvidos questionários, com base em roteiros semiestruturados, aplicados pelo autor, visando conhecer os agentes de interesse e motivação que os conduzirá ao tipo de aplicação financeira, objetivando ainda traçar o perfil do 
investidor e o conhecimento sobre o mercado de moedas eletrônicas. A outra parte dos dados foi obtida por meio de uma pesquisa quantitativa por meio da dados coletados no site da Ibovespa, Blockchain, Guia do bitcoin e coinmarketcap, observando a comparação entre investimentos de diferentes carteiras de ativos de ações, dentre elas as que apresentaram maior rentabilidade no ano de 2017, comparando-as com o bitcoin.

Decidiu-se observar o período de janeiro de 2015 a agosto de 2017, para 4 tipos diferentes de ativos, será utilizada como técnica o estudo da volatilidade através de um procedimento econométrico, envolvendo testes, entre eles o modelo autoregressivo $\operatorname{AR}(\mathrm{p})$, buscando-se o retorno através da média incondicional $\mathrm{AR}(\mathrm{p})$, e da variância incondicional $\operatorname{ARCH}(p)$ ou EGARCH (p, q). O teste DF-GLS foi utilizado para definir o número de passagens ótimas nos modelos $\mathrm{AR}(\mathrm{p}), \mathrm{ARCH}(\mathrm{p})$ ou EGARCH (p, q). Uma vez obtidas as médias incondicionais dos retornos e das variâncias, busca-se a analisar a significância estatística com a aplicação do teste T e do teste F.

\subsection{Tratamento econométrico}

Para avaliar a volatilidade dos ativos, estrutura-se um procedimento econométrico, envolvendo testes que visam verificar a existência de mudança estrutural para os ativos analisados, observando se os retornos e as variâncias dos ativos são diferentes ao longo do período estudado.

Os retornos dos ativos são obtidos por capitalização continua sendo:

$$
\mathrm{r} \mathfrak{t}=(\operatorname{In} A \mathfrak{t}-\operatorname{In} A \mathfrak{t}-1)
$$

onde $\mathrm{r}$ é o retorno do ativo, At é o ativo em pontos do período t; e At-1 é o ativo em pontos no período $\mathrm{t}-1$.

Um modelo autoregressivo AR(p) é utilizado para avaliar os parâmetros, conforme abaixo:

onde $\varepsilon \tau+1$ é o choque inesperado, tal que $\varepsilon \tau+1 \sim \mathrm{N}(0,1)$.

$$
r \tau=\alpha o+\sum_{p=1}^{n} \alpha \rho \mathfrak{R} \tau-p+\varepsilon \tau+1
$$

A média incondicional do modelo $\mathrm{AR}(\mathrm{p})$ é dada por:

$$
E(R \tau)=\frac{\alpha O}{\left(1-\sum_{p=1}^{n} \alpha \rho\right)} .
$$


O modelo ARCH(p) ou o modelo EGARCH(p) definido por Engle (1982) é utilizado para modelar a variância incondicional, dado pela equação:

$$
\sigma_{t}^{2}=\alpha o+\sum_{p=1}^{n} \alpha \rho a_{t-p}^{2}
$$

O modelo EGARCH $(1,1)$, foi apresentado por $\stackrel{t}{t}$ Nelson (1991) conforme abaixo:

$$
\operatorname{In}\left(\sigma_{t}^{2}\right)=y 0+\frac{\alpha 1 \alpha \tau-1+y(\alpha \tau-1)}{\sigma \tau-1}+\beta 1 \ln \left(\sigma_{t-1}^{2}\right)
$$

O cálculo da variância incondicional $\mathrm{ARCH}(\mathrm{p})$ é definida pela seguinte fórmula:

$$
E(R t)=\frac{y 0}{\left(1-\sum_{p=1}^{n} \alpha \rho\right)} .
$$

Para o modelo EGARCH (p, q), a variância incondicional e dada por:

$$
E(R t)=\frac{y o}{\left(1-\sum_{p=1}^{n} \beta \rho\right)} .
$$

O teste DF-GLS foi utilizado para definir dados referentes a defasagens ótimas do modelo $\operatorname{AR}(p), \operatorname{ARCH}(p)$ ou EGARCH (p, q). O teste DF-GLS é um teste eficaz de raiz unitária e é semelhante ao teste Dicky Fuller. Entretanto, o teste DF-GLS, segundo Elliot, Rothenberg; Stock, (1996), demonstra melhor representação ao interagir com os dados que apresentem média ou tendência desconhecidos.

Uma vez obtidas as médias incondicionais dos retornos e das variâncias, procura-se verificar o retorno dos ativos e a volatilidade, analisando ainda a significância estatística dos retornos através do teste t, empregado por Montgomery et al. (2016) como:

$$
t=\frac{\overline{X b}-\overline{X a}}{\sqrt{\frac{s \frac{b}{n b}}{n b}+\frac{s \frac{2}{n a}}{n a}}}
$$

sendo $\mathrm{X}$ o retorno do ativo, $\mathrm{S}$ a variância incondicional e n o número de observações.

Para verificar a ocorrência de mudanças na volatilidade das séries, realizou-se o teste de mudança de variância, definido por Montgomery et al. (2016) como:

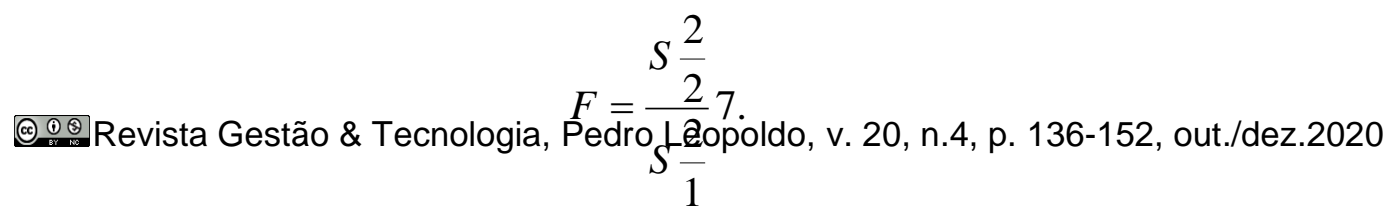




\section{RESULTADOS QUANTITATIVOS}

A tabela 1 apresenta os modelos indicados das informações regressivas e para os modelos da família GARCH. Desta forma, observa-se que as séries estão estimadas pelo modelo AR, sendo definida a partir da análise do correlograma das equações. Sendo AR(5) para o bitcoin, AR(1) MA(1) para o Itaú, AR(13) para a light e $\mathrm{AR}(5) \mathrm{MA}(5)$ para a Vale, conforme tabela 1 abaixo:

\begin{tabular}{lll} 
Tabela 1 & & \\
\multicolumn{3}{l}{ Modelo selecionado pelo DF-GLS } \\
\cline { 2 - 3 } INDICADOR & \multicolumn{1}{l}{ Modelo AR } & \multicolumn{1}{c}{ Volatilidade } \\
\hline BTC & AR(5) & ARCH(7) \\
& AR(1) & \\
Itaú & MA(1) & ARCH(7) \\
Light & AR(13) & EGARCH $(1,1)$ \\
& AR(5) & \\
Vale & MA(5) & ARCH(9) \\
\hline
\end{tabular}

Fonte: Elaborado pelos autores a partir de dados obtidos por meio do programa Eviews, 2020.

Ao analisar a tabela 2 abaixo, observa-se que em sua totalidade, os modelos estimados respeitaram a probabilidade estatística, obtendo valores inferiores a significância de 5\%, estimados pelo modelo AR(p). Os modelos de ARCH, EGARCH, também utilizados para estimar os coeficientes de volatilidade demonstraram significância de 5\% para o período analisado.

\section{Tabela 2}

Média incondicional do modelo AR(p) e variância incondicional dos modelos ARCH (p) ou EGARCH (p, q).

\begin{tabular}{llc}
\hline INDICADOR & \multicolumn{1}{c}{ MÉDIA } & VARIANCIA \\
\hline BTC & 0,00283540682 & 0,0045020664 \\
Itaú & 0,00024613567 & 0,0003837974 \\
Light & $-0,00036442580$ & 0,0009085759 \\
Vale & 7,52073915322 & 0,0010826429 \\
\hline
\end{tabular}

Fonte: Elaborado pelos autores a partir de dados obtidos por meio do programa Eviews, 2020.

Os resultados apresentados para as médias e as variâncias incondicionais demonstram o retorno próximo de zero para todos os indicadores, com exceção da Vale. O bitcoin 
apresentou maior variância, apresentando no inicio do ano de 2015 o valor de USD 314,89, alcançando o significante valor de USD 4.735,11 em agosto de 2017. Não obstante, a variação das ações da Vale, demonstram considerável significância. Os resultados revelam o preço da cotação em janeiro 2015 R \$22,29, fechando agosto de 2017 com o valor de R \$34,45. Embora a volatilidade do bitcoin seja a maior apresentada no período, não apresentou o maior retorno.

Observou-se que o aumento de utilitários da moeda bitcoin no Brasil ocorreu a partir da recessão apresentada no ano de 2015, entretanto; o aumento significativo de usuários ocorreu no primeiro e segundo semestre de 2017, o que ocasionou o aumento da procura pela moeda, consequentemente, contribuindo para uma supervalorização no mercado de moedas digitais, o que contribui para que a variância seja consideravelmente maior que os demais ativos analisados (Américo, 2017). Alguns fatores externos como a legalização da moeda em alguns países, possibilitando a utilização do bitcoin como forma legal de pagamento, contribuíram para a valorização da moeda.

\subsection{Teste da significância estatística dos dados}

Para avaliar de forma significativa os retornos dos ativos, gerados por meio da média incondicional, decidiu-se aplicar o teste estatístico $\mathrm{t}$ de student para a verificação das diferenças de médias. Os resultados encontrados na tabela 3 revelam os valores estimados. $\mathrm{O}$ teste verifica se os valores dos retornos são diferentes, devendo então ser interpretado como uma distribuição bicaudal.

A primeira coluna da tabela 3 testa a hipótese de que as séries de retornos analisados no bitcoin é estatisticamente diferente dos retornos observados para os demais ativos. Os resultados encontrados indicam que os retornos são estatisticamente significantes ao retorno observado em relação ao bitcoin. Os resultados $\mathrm{P}$ comparados através da tabela de distribuição do teste revelam que os retornos dos ativos Itaú e Light obtiveram o valor de $1 \%$ no quadro de distribuição P. O resultado de distribuição do ativo Vale revelou o resultado de $0,5 \%$. 
Tabela 3

Valor encontrado para o teste $\mathrm{T}$ de mudança de média incondicional

\begin{tabular}{lllll}
\hline Indicador & \multicolumn{1}{c}{ T-Btc } & \multicolumn{1}{c}{ T- Itaú } & \multicolumn{1}{c}{ T-Light } & \multicolumn{1}{c}{ T-Vale } \\
\hline Btc & & 1,159631201 & 1,36180802 & $3149,26^{* 1}$ \\
Itaú & $-1,159631201$ & & 0,531677698 & $-6147,90^{*}$ \\
Light & $-1,36180802$ & $-0,531677698$ & & $-5276,36^{*}$ \\
Vale & $3149,26^{*}$ & $6147,90^{*}$ & $5276,36^{*}$ & \\
\hline
\end{tabular}

Fonte: Elaborada pelos autores, 2020.

A segunda coluna demonstra a hipótese de que o retorno dos indicadores do Itaú é diferente dos demais ativos. Os resultados revelam que as ações apresentaram uma rentabilidade moderada, não havendo influencia demasiadamente alta tal que afetasse o comportamento do mercado para a manutenção da comercialização do ativo.

A terceira coluna testa a hipótese de que o retorno dos indicadores da Light é diferente dos demais ativos. A significância estatística revela-se nos ativos Btc e Itaú, demonstrando a forte influência do mercado para a valorização dos ativos no ano de 2017. O Btc alcançou uma alta histórica em sua precificação neste período e foi votado na Câmara dos Deputados o projeto de lei 2303/2015 que dispõe sobre a inclusão das moedas digitais na definição de arranjos de pagamento sob a supervisão do BACEN (Banco Central do Brasil), devido à grande demanda do mercado brasileiro pela moeda. Não obstante, a carteira de ativos do Itaú foi bem avaliada pelos analistas e pelas corretoras, alcançando maior número de investidores. Constata-se ainda que os retornos da Light se comparados com os demais ativos está entre o menor.

A última coluna testa a hipótese de que os retornos dos indicadores da Vale são diferentes dos demais ativos. Os resultados apresentados dos retornos da Vale revelaram uma rentabilidade superior as outras carteiras de ações. As variações sofridas nas cotações no período de 2015 a 2017 contribuíram para a concretização dos dados. Não obstante, os valores comparados através da distribuição do teste $\mathrm{P}$, revelam o valor superior ao retorno observado nas demais colunas, para um nível de significância $<$ ou $=1 \%$.

Ao analisar a tabela 4, apresentando o resultado para o teste de mudança de variância incondicional, observa-se que o valor da variância demonstra a influência do mercado para a movimentação dos ativos, o que ocasiona a volatilidade. A explicação para este tipo de ocorrência é que quanto maior for a variação dos preços dos ativos, maior será a volatilidade.

\footnotetext{
${ }^{1} \mathrm{O}$ asterisco significa o limite que tende a centralizar os valores em torno de zero, tal variação não afetará as casas decimais de forma a não comprometer as respostas.
} 
Na primeira coluna, verificou-se a variação do bitcoin em comparação com os demais ativos. Os resultados revelaram que o bitcoin apresentou a maior volatilidade dos ativos.

\section{Tabela 4}

Valor do teste $\mathrm{F}$ de mudança de variância incondicional

\begin{tabular}{lccll}
\hline INDICADOR & F-Btc & \multicolumn{1}{c}{ F- Itaú } & \multicolumn{1}{c}{ F-Light } & \multicolumn{1}{c}{ F-Vale } \\
\hline Btc & & 0,085249165 & 0,201813083 & 0,240476884 \\
Itaú & $11,73^{*}$ & - & $2,36^{*}$ & $2,82^{2}$ \\
Light & $4,955^{*}$ & 0,422416443 & - & $1,19^{*}$ \\
Vale & $4,15^{*}$ & 0,354500454 & 0,839220301 & - \\
\hline
\end{tabular}

Fonte: Elaborada pelos autores, 2020.

A segunda coluna da tabela 4 testa a hipótese de que a variância incondicional do Itaú é estatisticamente diferente dos demais ativos. O Itaú apresentou a menor volatilidade se comparado com o bitcoin. Os resultados revelaram uma volatilidade média em comparação com a Light e Vale, apresentando-se como o ativo com a menor variação.

A terceira coluna da tabela testa a hipótese da variância incondicional da Light. Os resultados indicam uma variação moderada diante dos outros ativos. Observa-se que a Light apresentou menor volatilidade se comparado com o ativo da Vale.

Por fim, a quarta coluna indica que a variância incondicional da Vale foi a que apresentou a maior volatilidade se comparado com o bitcoin, diferenciando-se das variâncias dos demais ativos. Observou-se ainda, que dentre as carteiras de ações, a Vale apresentou a maior variação.

Os resultados revelaram ainda que a diferenciação da variância dos ativos indica uma maior possibilidade de ganho ou perda. Sabe, porém, que o estudo da volatilidade compreende entender a capacidade de um ativo aumentar e diminuir seu valor e determinado fato ocorre muito no mercado financeiro, principalmente no mercado de ações. Para muitos, este cenário aparenta uma ótima oportunidade para lucrar, pois quanto maior o valor de um ativo, maior será o lucro com a sua venda. Entretanto, a volatilidade de um bem demonstra sua constante movimentação, inclusive facilitando a análise e verificação de oportunidades.

\footnotetext{
${ }^{2} \mathrm{O}$ asterisco significa o limite que tende a centralizar os valores em torno de zero, tal variação não afetará as casas decimais de forma a comprometer as respostas.
} 


\subsection{Análise dos dados coletados para o estudo}

Nesta seção se apresenta a análise dos dados obtidos a partir da análise das respostas por meio da aplicação de um questionário com perguntas direcionadas ao tema. A pesquisa contou com a participação de 80 pessoas, sendo 40 participantes do marketing de rede de uma empresa mineradora de bitcoin. Os demais 40 participantes se constituíram de empresários, investidores, economistas, gerentes e universitários, representando pessoas da população.

Os participantes foram questionados sobre como consideravam um investimento com bitcoin e se o bitcoin seria um investimento de alto risco. Dentre os 80 participantes, verificou-se que apenas 25 responderam que consideram fazer investimentos em bitcoin, correspondendo a $31,25 \%$ das pessoas entrevistadas, pois acreditam que o bitcoin é um investimento de risco. As outras 55 pessoas participantes da pesquisa que correspondem a $68,75 \%, 40$ são integrantes do mercado bitcoin, sendo que apenas 15 pessoas que não atuam neste ramo de investimentos de moedas eletrônicas, compartilham do mesmo pensamento dos demais.

A questão número 2, visava avaliar o conhecimento dos envolvidos em aplicações financeiras. Para isto, foi observado o grau de conhecimento dos avaliados quanto a fundos de renda fixa, fundos multimercados, fundos de ações, fundos de crédito. Observou-se o grau de conhecimento classificado como alto, médio, baixo e nenhum.

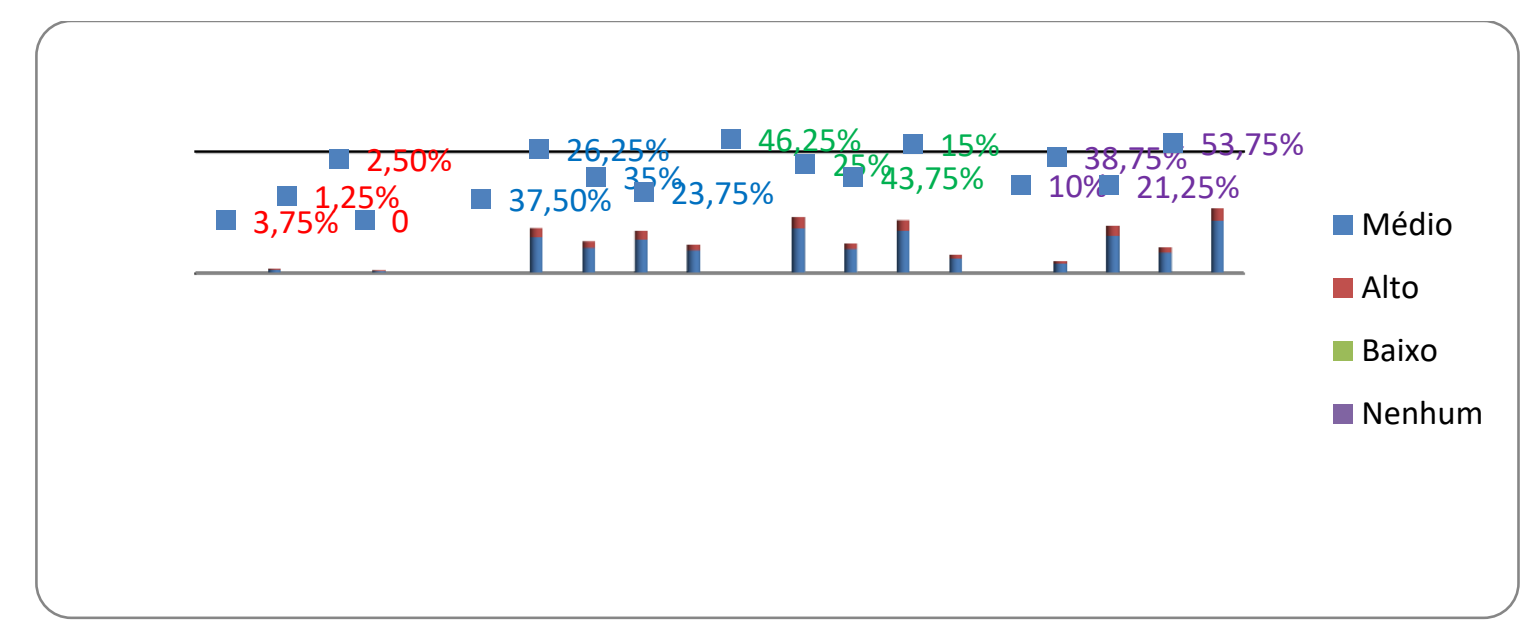

Figura 1 - Classificação das respostas sobre conhecimento em aplicações Fonte: Elaborada pelos autores, 2020.

Verificou-se nos resultados que poucos demonstraram um conhecimento alto sobre fundos de renda fixa, indicando 3 respostas, que corresponde a 3,75\%. Por outro lado, 
verificou-se que 37 pessoas responderam que tem um baixo conhecimento sobre renda fixa, o que corresponde a $46,25 \%$.

Os resultados apresentam uma minoria da população com alto conhecimento em investimentos. Nota-se ainda os maiores resultados contidos entre os índices baixo e médio. Entretanto, os dados da pesquisa revelam ainda que 53 pessoas já tiveram ou tem uma aplicação em renda fixa, o que corresponde a $66,25 \%$.

\section{Tabela 5}

Tipos de investimentos dos entrevistados

\begin{tabular}{lc}
\hline Poupança & $88,75 \%$ \\
Títulos de renda & $6,25 \%$ \\
Títulos públicos & $3,75 \%$ \\
Fundos de renda fixa & $66,25 \%$ \\
Fundos multimercado & $16,25 \%$ \\
Fundos de ações & $21,25 \%$ \\
Fundos imobiliários & $7,50 \%$ \\
Fundos de previdência privada & $36,25 \%$ \\
Ações & $7,50 \%$ \\
Mercado futuro (BM\&F) & 0 \\
Bitcoin & $50 \%$ \\
Nunca tive investimentos & $5 \%$ \\
\hline
\end{tabular}

Fonte: Elaborada pelos autores, 2020.

Os resultados apresentados para investidores na poupança foram os maiores, sendo o total de 71 respostas, correspondendo a 88,75\% conforme a tabela 5. Os valores apresentados, para o índice de renda fixa, confirmaram as pesquisas apresentadas pelo Tesouro Nacional (2017), onde se constatou o grande aumento de venda de títulos com vencimentos entre 5 e 10 anos e o crescimento de investidores ativos em 12.759 no mês de março/2017, obtendo uma variação de $91,9 \%$ nos últimos doze meses.

Os resultados confirmam a busca por maior segurança em investimentos, dado a recuperação econômica no segundo semestre de 2017. Por outro lado, os investimentos em renda variada não demonstram grande interesse. Os resultados anteriores demonstraram o baixo conhecimento dos entrevistados neste tipo de aplicação. Entretanto, há grande público com perfil para este tipo de investimentos mais agressivos e arriscados, como no caso do bitcoin. 
Observou-se que os participantes de investimentos em bitcoin têm idade variada, entretanto, são na maioria de 27 a 44 anos, levando-se em consideração a dificuldade das pessoas com mais de 47 anos, em lidarem com este tipo de tecnologia.

Algumas instituições financeiras traçam um perfil para seus investidores. Segundo Luis (2016), o investimento deverá ser de acordo com o objetivo a ser alcançado, a situação financeira e o conhecimento do investidor. Desta forma, não se torna difícil identificar o perfil de um investidor de bitcoin. Para os dados acima, verifica-se que para os 40 participantes da rede bitcoin, todos participaram ou participam de outro de investimento.

Entretanto, a parte da população investidora de ativos financeiros de renda variável ainda se constitui de uma minoria. Tal questão pode ser representada na pesquisa onde no total de 80 entrevistados, apenas 6 tiveram ou tem aplicação em ações, o que corresponde a $7,50 \%$ dos entrevistados. Quanto aos investimentos em bitcoin, apenas os 40 participantes da rede de relacionamento da empresa mineradora tiveram ou tem este tipo de aplicação.

Por fim, constatou-se que todos os envolvidos em aplicações com bitcoin consideram a moeda como um investimento seguro e altamente rentável.

\section{CONSIDERAÇÕES FINAIS}

Nos últimos anos houve um grande interesse da população brasileira e dos demais países para o investimento em bitcoin. Tal fato pode ser explicado por meio da oferta e demanda da moeda, o que ocasionou à alta do valor cotado da moeda, apresentando um crescimento alarmante de usuários no ano de 2017, despertando a curiosidade dos bancos, Estados e investidores.

Entretanto, este fator despertou a preocupação dos analistas e do mercado devido sua volatilidade. Ao serem analisados outros ativos e compará-los com o bitcoin, verificou- se que todo ativo de renda variável está passível de volatilidade devido à inconstâncias do mercado. Constatou-se no bitcoin, uma maior volatilidade e rentabilidade em comparação com outros quatro ativos de renda variável estudados.

Como o objetivo proposto no estudo foi o de verificar a rentabilidade e a volatilidade da moeda, considera-se que o estudo teve êxito por meio do tratamento econométrico sugerido, observando-se os retornos e as variâncias dos ativos ao longo do período estudado. Os resultados alcançados demonstraram que o bitcoin foi o ativo com maior volatilidade, em comparação a outros três ativos, de diferentes carteiras de ações, que apresentaram os maiores 
rendimentos no ano de 2017. Entretanto, quanto mais volátil um ativo, maior a sua capacidade de aumentar e diminuir de valor.

Portanto, os resultados apresentados por meio da pesquisa de campo revelaram a preocupação dos indivíduos envolvidos na pesquisa em se ter uma aplicação segura. Demonstraram, ainda, o pouco conhecimento em relação a títulos de renda variável. Verificou-se a satisfação dos indivíduos envolvidos no mercado de moedas eletrônicas com um perfil agressivo que visa alta rentabilidade.

\section{REFERÊNCIAS}

AMÉRICO, F. (2017). BOLSA DE AÇÕES AUSTRALIANA PASSA A USAR BLOCKCHAIN PARA LIQUIDAR NEGÓCIOS. GUIA DO BITCOIN. 07/12/2017. DISPONÍVEL EM: $<$ https://guiadobitcoin.com.br/bolsa-de-acoes-australiana-passa-a-usar-blockchain-paraliquidar-negocios/>. Acesso em: 10 dez. 2017.

Banco Central do Brasil. (2017). Mais de $70 \%$ dos brasileiros utilizam contas bancárias, segundo pesquisa do BC. 24/10/2016. Disponível em: <http://www.bcb.gov.br/ptbr/\#!/c/noticias/33>. Acesso em: 16 out. 2017.

Barreiros, M. R. (2016). Bitcoin: Uma moeda para a era digital. Lisboa, dezembro, 2016. Dissertação (Mestrado em Estratégia de Investimento e Internacionalização), business \& economics school, Lisboa, Portugal.

Bourdeaux, R. R.; Ness JR., W. L. (2016). A Preferência por Subscrições Privadas de Ações no Brasil. RAE Eletrônica, v. 6, n. 2, p. 15-29.

Correia, B.S.; Alencar, L.F.M.; Amilton P.M.L.F. (2017). Análise da relação entre os retornos do bitcoin e os fatores de risco: uma abordagem multifatorial. Trabalho apresentado no XI congresso ANPCONT, Belo Horizonte.

Cryptocurrency Market Capitalizations. (2017). All cryptocurrencies: full list. Disponível em: https://coinmarketcap.com/all/views/all/. Acesso em: 10 dez. 2017.

Elliott, G.; Rothenberg, T. J.; Stock, H. J. (1996). Efficient tests for an autoregressive unit root. Econometrica, v. 64 (4, Jul), 813-836.

Engle, R. F. (1992). Autoregressive conditional heteroscedasticity with estimates of the variance of United Kingdom inflation. Econometrica: Journal of the Econometric Society, 987-1007.

Eviews (2017). Innovative solutions for econometric analysis, forecasting e simulation. Disponivel em: <http://www.eviews.com/home.html>. Acesso em: 16 out. 2017.

Luis, R. S. F. (2016). Otimização dos investimentos para pessoa física no Brasil através do modelo de portfólio de markowitz. Criciúma: SC, 2016. Dissertação (Bacharel em ciências econômicas), Universidade do Extremo Sul Catarinense, Criciúma: SC.

Mesquita, D. B. (2017). Moeda e criptomoeda: uma análise do bitcoin sobre a perspectiva pós-keynesiana. Florianópolis: SC, 2017. Dissertação (Bacharel em ciências econômicas), Universidade Federal de Santa Catarina, Florianópolis: SC.

Mesquita, S. B. M. M. (2017). O bitcoin e seu impacto para a sociedade e para o setor financeiro. Palhoça: SC, 2017. Dissertação de graduação (Bacharel em ciências econômicas), Universidade do Sul de Santa Catarina, Palhoça: SC. 
Markovitz, H. M. (1952). Portfolio selection. The Journal of Finance, v. 7, n.1, p. 77-91.

Montgomery, D. C.; Runger, G. C. (2016). Estatística aplicada e probabilidade para engenheiros. Livros Técnicos e Científicos. Rio de Janeiro: LTC.

Nelson, D. B. (1991). Conditional heteroskedasticity in asset returns: A new approach. Econometrica: Journal of the Econometric Society, 347-370, 1991.

Oliveira, C. Reportagem bitcoin. Jornal Hoje 29/11/2017. Youtube. 29 nov. 2017. Disponível em: 〈https://www.youtube.com/watch?v=rH4eLgRQJW4>. Acesso em: 12 dez. 2017.

Tesouro Nacional. (2017). Tesouro Direto bate recorde de novos investidores. Disponível em: <http://www.tesouro.gov.br/web/stn/-/tesouro-direto-bate-recorde-de-novos-investidores>. Acesso em: 16 out. 2017. 